\title{
Cancellation of Global Anomalies in Spontaneously Broken Gauge Theories
}

\author{
M. Fabbrichesi, R. Percacci, M. Piai, and M. Serone \\ INFN, Sezione di Trieste and \\ Scuola Internazionale Superiore di Studi Avanzati \\ via Beirut 4, I-34014 Trieste, Italy.
}

(Dated: October 30, 2018)

\begin{abstract}
We discuss the generalization to global gauge anomalies of the familiar procedure for the cancellation of local gauge anomalies in effective theories of spontaneously broken symmetries. We illustrate this mechanism in a recently proposed six-dimensional extension of the standard model.

PACS numbers: $11.10 . \mathrm{Kk}, 11.15 . \mathrm{Ex}, 11.30 .-\mathrm{j}$
\end{abstract}

\section{INTRODUCTION}

It is always possible to cancel gauge anomalies in effective theories by introducing non-renormalizable operators. These operators contain boson fields that transform non-linearly under the corresponding gauge symmetries. These fields can either be introduced as additional degrees of freedom - as in the Green-Schwarz (GS) [1] and other mechanisms [2] - or be the would-be Goldstone bosons of the spontaneous breaking of the gauge symmetry 3 .

After briefly reviewing the literature on local (perturbative) gauge anomalies, we discuss in some detail the global (non-perturbative) case [4] in $d$-dimensional effective theories of a gauge group $G$ spontaneously broken to $H$. We show how to construct local operators that cancel both local and global anomalies, using the wouldbe Goldstone bosons of the spontaneously broken theory without introducing additional degrees of freedom. This can be done provided that: ${ }^{1}$

1. the coset space $G / H$ is reductive;

2. the fermion representations are free of local anomalies when restricted to the group $H$;

3. the fermion representations are free of global anomalies when restricted to the group $H$;

4. $G$ can be embedded in a group $K$ such that its homotopy group $\pi_{d}(K)=0$ and the fermion representations can be extended to $K$ without generating further anomalies of $G$.

Most of these conditions can be derived from the literature. In particular, conditions 1 and 2 have been derived for local gauge anomalies [5, 6, 7], while conditions 3 and 4 are closely related to the results of [8, 9, 10]. In this paper - generalizing the idea of computing global anomalies as local anomalies of a larger group $K[10]$-we argue that the operators constructed in [5, 6, 7] to cancel local

\footnotetext{
1 A possible limitation of this procedure arises whenever we wish to preserve further symmetries of the original action that are explicitly broken by the introduction of these operators.
}

anomalies of $G$ can be properly defined globally and used to cancel global anomalies of $G$, provided the above four conditions are satisfied.

This result is of interest in model building in so far as the fermion content of an effective theory is only restricted by the cancellation of the anomalies of the unbroken group $H$. As an example we show how the standard model in $d=6$ [11] can be made (local and global) anomaly free.

\section{CANCELLATION OF LOCAL GAUGE ANOMALIES}

In purely bosonic low-energy effective actions, anomalies are accounted for by the Wess-Zumino (WZ) term [12]. For a global symmetry group $G$ spontaneously broken to an anomaly-free subgroup $H$, the WZ coupling is a non-invariant function of the corresponding Goldstone bosons. The same term can be generalized to reproduce also gauge anomalies when part or all of the group $G$ is gauged [8]. From an effective field theory point of view, the WZ term can be seen as the remnant of massive fermions that have been integrated out in a microscopic theory [13]. This is in agreement with the 't Hooft anomaly matching condition [14]: anomalies, being long-distance effects, have to match in going from the fundamental to the effective theory.

The WZ term can also be viewed from a different perspective: an anomalous theory can be rendered gauge invariant by coupling it to $G$-valued scalar fields and adding the WZ term to the action [2. Thus, instead of reproducing the anomalies of an underlying theory, one can use the WZ term to cancel them. This construction is similar to the GS mechanism [1], which also requires the presence of additional degrees of freedom (but only works for reducible anomalies).

If $\Gamma(A)$ is the effective action for the gauge field $A$ obtained by integrating out fermions, the WZ term is defined in general by

$$
\Gamma_{W Z}(A, g)=\Gamma\left(A^{g}\right)-\Gamma(A),
$$

where $A^{g}=g^{-1} A g+g^{-1} d g$ is the gauge transformed connection. From the definition, it satisfies the following 
(cocycle) condition

$$
\Gamma_{W Z}\left(A^{g}, U^{g}\right)-\Gamma_{W Z}(A, U)=-\Gamma_{W Z}(A, g),
$$

where $U$ are the $G$-valued scalar fields and $U^{g}=g^{-1} U$. If the low-energy theory contains both fermions and scalars, and the scalar action contains the WZ term, then the effective action $\tilde{\Gamma}(A, U)=\Gamma(A)+\Gamma_{W Z}(A, U)$ is seen to be gauge invariant by means of (11) and (2). It can be shown that the addition of the WZ term cures also the problems arising in the canonical formulation of the theory [18], so that the quantum theory, aside from renormalizability issues, is well defined.

Although the fermionic effective action is nonlocal, the $\mathrm{WZ}$ action is local. The standard way of deriving an explicit expression for the WZ action is through the dimensional descent. Assume that the boundary conditions on the fields are such that spacetime can be compactified to a $d$-dimensional sphere (we assume $d$ to be even). We can think of this sphere as the boundary of a $d+1$-dimensional ball $B$. Assume for simplicity that the gauge fields are topologically trivial (no instantons) and that the scalar fields are homotopically trivial. (This will automatically be the case if the homotopy group $\pi_{d}(G)=0$, but we need not assume this). Then the fields can be extended to a gauge field $\hat{A}$ and a scalar field $\hat{U}$ defined on all of $B$. Let $\Omega(\hat{A})$ be the Chern-Simons functional, the integral of the $(d+1)$-form $\omega(\hat{A})$ on $B$. Its gauge variation under a gauge transformation $g$ is the WZ action:

$$
\Gamma_{W Z}(A, g)=\Omega\left(\hat{A}^{\hat{g}}\right)-\Omega(\hat{A}) .
$$

Even though the right-hand side of (3) is written in terms of the fields on $B$, it is the integral of an exact form and therefore can be rewritten as an integral on spacetime, depending only on the boundary values of the fields $\hat{A}$ and $\hat{U}$. This gives an explicit, local formula for the $\mathrm{WZ}$ term that cancels local anomalies.

A variation of this anomaly-cancellation scheme occurs when the scalar fields are not introduced by hand but are already present in the theory. This can happen in the Higgs phase of a gauge theory [3]. The wouldbe Goldstone bosons - the angular components of the Higgs field - are the scalar fields that can be used to write the WZ term. This idea had been already put forward in [15] where the interplay between renormalizability and anomaly cancellation in spontaneously broken gauge theories was first discussed in an abelian Higgs model coupled to a chiral fermion in four dimensions. This perspective - that spontaneously broken effective gauge theories with anomalous fermion content can be consistently quantized - has been exploited in [3] where the approach is extended to non-abelian gauge symmetries.

Since there is usually a nontrivial unbroken group $H$, one has to generalize the WZ term to the case of $G / H$ valued, rather than $G$-valued, scalars $\varphi$. Let us review more explicitly how this construction works, following closely the analysis in [5, 6, 7] from the perspective of [3].
Consider a given $d$-dimensional action with a local gauge symmetry $G$ spontaneously broken to a subgroup $H$. We denote by $T^{A}$ the whole set of generators of the Lie algebra $\mathcal{G}$ of $G$, whereas $T^{i}$ and $T^{\alpha}$ are respectively the generators of $\mathcal{H}$ and of the coset $G / H$. We assume that $G / H$ is a reductive space, i.e. that $\left[T^{i}, T^{\alpha}\right]=f_{i \alpha \beta} T^{\beta}$. Assume also that the fermion content of the corresponding action gives rise to the following anomaly:

$$
\begin{aligned}
\delta_{\alpha} \Gamma(A) & =\int d^{d} x \mathcal{A}_{\alpha}[A(x)], \\
\delta_{i} \Gamma(A) & =0,
\end{aligned}
$$

where $\mathcal{A}_{\alpha}$ denotes the usual one-loop gauge anomaly. In other words, eq.(1) implies that any potentially anomalous fermionic one-loop amplitude vanishes as soon as one of the external gauge fields belongs to $H$.

To write a WZ term, we now assume that there exists a unitary gauge, i.e. that there is a globally defined gauge transformation $U(x)$ that transforms the $G / H$ valued field $\varphi(x)$ to a constant. ${ }^{2}$ This is a purely topological restriction on $\varphi$; it is less restrictive than the assumption of being homotopic to a constant.

This gauge transformation $U$ is not unique: two $G$ valued maps $U$ and $U^{\prime}$ correspond to the same $\varphi$ if and only if they differ by a right- $H$ transformation: $U^{\prime}(x)=U(x) h(x)$. Thus we can use $U$ as a dynamical variable instead of $\varphi$, but in doing so, we introduce an additional $H$ gauge freedom. Having reformulated the theory in terms of $U$, we can add to the action a WZ term $\Gamma_{W Z}(A, U)$. Because of (4), $\Gamma_{W Z}(A, U h)=\Gamma_{W Z}(A, U)$, so it depends only on the coset $\varphi(x)=U(x) H$, i.e. on the would-be Goldstone fields. In this case we will therefore write $\Gamma_{W Z}(A, \varphi)=\Gamma_{W Z}(A, U)$. The addition of this term to the action cancels the anomaly exactly as in the previous case. ${ }^{3}$

This procedure can be used also when the second condition in eq. (A) is replaced by the weaker condition $\delta_{i} \Gamma\left(A_{H}\right)=0$ where $A_{H}$ denotes the component of the gauge field in the subalgebra of $H$, i.e. when the fermion representations, restricted to the subgroup $H$, are free of local anomalies. As shown in 5, 6, 7], one can add to the action a local functional $B_{d}\left(A_{H}, A\right)$ such that the second relation in (4) is satisfied (see [7] and eq. (12) below for an explicit expression of $\left.B_{d}\left(A_{H}, A\right)\right)$. Thus, in this more general setting, all local anomalies are cancelled by

\footnotetext{
2 This is always true whenever the gauge field $A$ and its components in $H$ are topologically trivial. More general cases can be however similarly analyzed.

${ }^{3}$ One often encounters the notation $U(x)=e^{\xi^{\alpha}(x) T_{\alpha}}$; this amounts to choosing a specific coordinate system on the coset space and can generally be valid only locally. In this notation $\Gamma_{W Z}(A, \xi)=-\int_{0}^{1} d t \int d^{d} x \xi^{\alpha}(x) \mathcal{A}_{\alpha}\left[A_{t}\right]$ where $A_{t}=e^{-t \xi} A e^{t \xi}+$ $e^{-t \xi} d e^{t \xi}$. In the simple case of $G=U(1)$ and $H=\{1\}$ one can easily check that this reduces to the form $\int \xi F \tilde{F}$, 15. as also discussed in 16 in the context of the GS mechanism in $d=4$.
} 
adding to the action the modified WZ term

$$
\Gamma_{W Z}^{\prime}(A, U)=\Gamma^{\prime}\left(A^{U}\right)-\Gamma^{\prime}(A),
$$

where

$$
\Gamma^{\prime}(A)=\Gamma(A)+B_{d}\left(A_{H}, A\right) .
$$

Once gauge invariance is restored, it is possible to shift the fields in such a way as to decouple $\varphi$, and give mass to the gauge bosons of $G / H$ (unitary gauge). The presence of the term (5) in the generic gauge signals however the non-renormalizability of the theory. An explicit proof of non-renormalizability in the 't Hooft-Landau gauge is given in [3]. This can also be understood by looking at the diagram giving rise to the anomaly containing offshell non-analytic contributions 17] that cannot be cancelled by any local counterterm.

The result of these analyses is that, as far as local gauge anomalies are concerned, effective theories, for which renormalizability is not a requirement, can be made anomaly free by the addition of an appropriate WZ term. No restriction on the fermion content of the theory is needed, provided that the first two conditions listed in the introduction are satisfied.

So far, our analysis has been purely local without considering possible topological obstructions or possible global gauge anomalies. In fact, a potential problem can arise in the above construction whenever $\pi_{d}(G / H) \neq 0$ [7]. On the other hand, the same condition of having a non-trivial homotopy group can give rise to global anomalies [4]. In the next section we will show how to generalize the above procedure to take into account these global issues and remove the above topological condition.

\section{CANCELLATION OF GLOBAL GAUGE ANOMALIES}

Even a theory free of perturbative anomalies - i.e. invariant under infinitesimal gauge transformations - can still be anomalous under gauge transformations that are not homotopic to the identity. This was discussed first for an $S U(2)$ theory in four dimensions in [4] and for other dimensions in [10] (see also 19]). These gauge anomalies are called global, or non-perturbative anomalies. They can occur whenever $\pi_{d}(G) \neq 0 .{ }^{4}$

Extending the results of [2], it is always possible to cancel any global anomaly by coupling the theory to a $G$ valued scalar field $U(x)$ and adding a suitable WZ term $\gamma$ to the action 20]. The absence of local anomalies means that $\gamma$, defined as in (1D), is zero, independently of $A$, when $g$ is homotopic to a constant. Every $g$ in a certain

\footnotetext{
4 When spacetime is not flat, or instantons are present, the conditions can become more complicated. We will not consider these cases.
}

homotopy class can be written as $g_{1} g^{\prime}$ where $g_{1}$ is a fixed map in that same homotopy class and $g^{\prime}$ is homotopically trivial. Then from the cocycle condition (2) one sees that $\gamma\left(A, g_{1} g\right)=\gamma\left(A, g_{1}\right)$, so $\gamma$ is invariant under continuous deformations of $g$. In conclusion, $\gamma$ depends only on the homotopy class $[g]$ and therefore can be seen as a topological term. ${ }^{5}$ From (2) we also see that it must define a representation of $\pi_{d}(G)$ :

$$
\gamma\left(g_{1} \cdot g_{2}\right)=\gamma\left(g_{1}\right)+\gamma\left(g_{2}\right) .
$$

As in section II, we are especially interested in the case where the scalar fields do not have to be introduced ad hoc, but are already present in the theory. We are thus led to ask: if the $G$ gauge symmetry is spontaneously broken to $H$ due to a Higgs mechanism, can one cancel the global anomalies of the low-energy effective theory by means of a WZ term written as a functional of the would-be Goldstone bosons? The answer is that this is possible if the fermion representations, restricted to $H$, are free of global anomalies.

As in the previous section we replace the coset-valued field $\varphi$ by the $G$-valued field $U$. Having reformulated the theory in terms of $U$, we can write WZ terms $\gamma(U)$ cancelling any global anomaly, using the method described above. Of course now we want these terms to depend only on the physical scalar fields $\varphi$. This will automatically be the case if the unbroken group $H$ is free of global anomalies. Indeed, in this case it follows from (7) that $\gamma(U \cdot h)=\gamma(U)+\gamma(h)=\gamma(U)$ and therefore $\gamma$ really only depends on the (homotopy class of the) coset-valued field $\varphi(x)$. Global $H$ anomalies will certainly be absent if $\pi_{d}(H)=0$, but even if $\pi_{d}(H)$ is nontrivial the theory may be free of global $H$ anomalies provided the fermion representations are chosen appropriately. In this case, global anomalies can be present only when $\pi_{d}(G / H) \neq 0$.

One may be interested in a general method for calculating the topological term $\gamma$. Following the discussion in [9] and [10], it can be written as a WZ term, albeit for a larger group. The construction of the WZ term given in (3) demands that the map $U$ be homotopic to a constant. If $U$ is not homotopic to a constant one can still proceed by embedding $G$ in a larger group $K$ such that $\pi_{d}(K)=0$. One then defines a map $\bar{U}$ by composing $U$ with this embedding, and a gauge field $\bar{A}$ by the corresponding embedding of the Lie algebras. For instance, one can write [8]

$$
\bar{U}=\left(\begin{array}{cc}
U & 0 \\
0 & 1
\end{array}\right) \quad \text { and } \quad \bar{A}=\left(\begin{array}{cc}
A & 0 \\
0 & 0
\end{array}\right) .
$$

The fermion content of $K$ is so chosen that upon reduction to $G$ gives rise to the required $G$ representations and

\footnotetext{
5 The addition of a topological term to the action is a hallmark of the existence of $\theta$-sectors, and indeed one can interpret this anomaly-cancellation mechanism by saying that the anomalous theory can be quantized by coupling it to a specific $\theta$-sector of the scalar theory.
} 
a number of $G$ singlets (or anomaly-free representations of $G)$.

The field $\bar{U}$ is homotopic to a constant and one can explicitly write the WZ term $\Gamma_{W Z}^{K}(\bar{A}, \bar{U})$ as in (3). As described above, if the theory is free of perturbative anomalies we can take simply $\gamma(g)=\Gamma_{W Z}^{K}(\bar{A}, \bar{g})$. Thus, the WZ term canceling the nonperturbative $G$-anomaly can be calculated as a perturbative $\mathrm{WZ}$ term for the larger group $K$, along the lines of $[10]$.

Finally, let us consider the generic case when the theory has perturbative anomalies with only $\delta_{i} \Gamma\left(A_{H}\right)=0$, and at the same time $\pi_{d}(G / H) \neq 0$. In the last section we have seen, following [5, 6, 7], that it is always possible to add to the action a local operator $B_{d}\left(A_{H}, A\right)$ so as to construct a WZ term (5) and cancel the perturbative anomalies. This WZ term is well defined when the map $U$ is homotopic to a constant. We have also described in the previous paragraph a way of writing a WZ term for a larger group $K$ that, when restricted to the subgroup $G$, makes sense for all maps $U$, irrespective of their homotopy class. If there are local anomalies, this WZ term, now denoted $\Gamma_{W Z}^{\prime K}(\bar{A}, \bar{U})$, is no longer topological. In fact, one can see (e. g. , by means of (8)) that when $U$ is homotopically trivial, it agrees with $\Gamma_{W Z}^{\prime}(A, U)$ defined in $(5)$. There follows that defining $\Gamma_{W Z}^{\prime}(A, U)=\Gamma_{W Z}^{\prime K}(\bar{A}, U)$ the resulting effective action is defined for all maps $U$, whether trivial or not, and free of both local and global gauge anomalies for the group $G$ spontaneously broken to $H .{ }^{6}$

A physical interpretation of the group $K$ can be given (although not necessary), along the lines of [13. In this case, one can imagine a microscopic theory with a gauge group $K$ and a completely anomaly free (local and global) fermion spectrum. The fermions are in representations of $K$ such that, upon the spontaneous breaking of $K$ to $G$, give rise to the required fermions in representations of $G$. The massive fermions that are integrated out produce the Wess-Zumino term $\Gamma_{W Z}^{\prime K}(\bar{A}, \bar{U})$ above.

\section{THE STANDARD MODEL IN SIX DIMENSIONS}

In a recent paper 11], it was argued that, in the minimal non-supersymmetric version of the standard model in $d=6$ dimensions, the requirement of canceling all gauge anomalies restricts the chiral fermion content of the theory. In particular, the cancellation of the $S U(2)$ global anomaly implies a restriction on the number $N_{g}$ of matter families:

$$
N_{g}=0 \bmod 3 .
$$

The field content of a single family is restricted by

\footnotetext{
${ }^{6}$ Even in the absence of global anomalies, this construction generalizes that of 1 .
}

the requirement of the cancellation of all irreducible local anomalies - that is, by the requirement of having a vector-like theory of strong and gravitational interactions - while the $d=6$ realization of the usual GS mechanism is invokated in order to eliminate reducible local anomalies. Accordingly, the fields are those in Table 4

TABLE I: Fermionic field content for each family in [11. The six-dimensional chirality is the eigenvalue of $\Gamma_{7}$. Here we put just one representative of the allowed combinations of chiralities; other choices are equivalent as far as the present discussion is concerned.

\begin{tabular}{|c|c|c|c|c|}
\hline \hline & Chirality & $U(1)_{Y}$ & $S U(2)_{L}$ & $S U(3)_{c}$ \\
\hline$Q$ & + & $1 / 6$ & 2 & 3 \\
$U$ & - & $2 / 3$ & 1 & 3 \\
$D$ & - & $-1 / 3$ & 1 & 3 \\
$L$ & + & $-1 / 2$ & 2 & 1 \\
$E$ & - & -1 & 1 & 1 \\
$N$ & - & 0 & 1 & 1 \\
\hline \hline
\end{tabular}

A detailed inspection of the GS mechanism, performed in [21], showed that the addition of two GS 2-formssupplemented by the use of the $U(1)_{Y}$ Goldstone boson in order to realize the generalized abelian GS of [16]-is sufficient to restore gauge invariance. After compactification of the two extra-dimensions, some pseudo-scalar fields, behaving like Peccei-Quinn axions, remain in the $d=4$ effective field theory as remnants of the GS fields. Their presence solves the strong $C P$ problem, but imposes a strong bound on the scale of the GS couplings and therefore a limit on the volume of the compact extradimensions, the fundamental scale of which turns out to be in the range of usual GUT theories.

Is it possible to achieve the cancellation of the reducible anomalies with some mechanism which does not leave any axionic remnant in the low energy $d=4$ effective theory? The machinery discussed in this paper is suitable for this purpose.

The choice of fermion content guarantees that the model has no pure gravitational anomaly. The addition of a local Chern-Simons term shifts mixed gravitational anomalies into those of gauge. Fermions form a vectorlike representation of the group $S U(3)_{c}$ with no contribution to the anomalies. We can thus identify the groups of the previous sections with those in [11]

$$
\left\{\begin{array}{l}
K=S U(4)_{L} \times U(1)_{Y} \\
G=S U(2)_{L} \times U(1)_{Y} \\
H=U(1)_{\text {e.m. }}
\end{array}\right.
$$

Since $\pi_{6}(G / H)=\mathbf{Z}_{12}$, we have enlarged $G$ to $K$ for which $\pi_{6}(K)=0$.

Each $S U(2)$ doublet in Table II goes into the fundamental representation of $S U(4)$ (to be decomposed into a 
$S U(2)$ doublet plus singlets). In addition to the alreadypresent singlets, more singlets are necessary in order to preserve the $U(1)_{Y}^{4}$ anomaly.

The subgroup $H$ is not anomaly free, but it is a simple exercise to verify that, with the field content in Table If, fermions form a non-chiral representation of the unbroken group $H$ : the weaker condition leading to the $B_{6}$ term is satisfied. Also all the other conditions stated in the introduction hold, and therefore the WZ term can be used to cancel all anomalies, as explained in the previous sections. This term can be explicitly written as

$$
\Gamma_{W Z}^{\prime K}=-\int_{0}^{1} \mathrm{~d} t \xi^{\alpha} \mathcal{A}_{\alpha}^{\prime}\left[\bar{A}_{t}\right]
$$

where $\Gamma^{\prime}(A)=\Gamma(A)+B_{6}\left(A_{H}, A\right)$, with

$$
B_{6}\left(A_{0}, A_{1}\right)=12 \int_{\Delta} \mathrm{d} \mu \mathrm{d} \lambda \operatorname{Str}\left[A_{0}, A_{1}, F_{\mu, \lambda}^{2}\right],
$$

and $A_{t}$ is defined in footnote 3 . Following the notation of [7]:

$$
\begin{aligned}
\operatorname{Str}\left[C_{1}, \cdots, C_{N}\right] & =\sum_{P} \frac{(-1)^{f_{P}}}{N !} \operatorname{Tr}\left[C_{P_{1}} \cdots C_{P_{N}}\right], \\
\mathcal{A}_{\alpha}^{\prime}\left(A_{t}\right) & =\mathcal{A}_{\alpha}\left(A_{t}\right)+\delta_{\alpha} B_{6}\left(A_{H}, A\right), \\
F_{\mu, \lambda} & =\mathrm{d} A_{\mu, \lambda}+A_{\mu, \lambda}^{2}, \\
A_{\mu, \lambda} & =\mu A_{0}+\lambda A_{1} .
\end{aligned}
$$

In eq. (11), the fields $\xi^{\alpha}$ are the longitudinal components of the massive gauge bosons $W$ and $Z$. $\mathcal{A}_{\alpha}$ is the (one-loop) anomaly. All fields must be thought as $K$ valued, as in (8). The integration region $\Delta$ is a triangle in the $(\mu, \lambda)$ plane with vertices $(0,1),(1,0)$ and the origin; $f_{P}$ is the number of times the permutation $P$ permutes two odd objects.

Thanks to this construction, no GS fields are needed; since there are no axions in the low energy $d=4$ theory, their experimental bounds do not apply. However, all global anomalies are canceled as well, and therefore the interesting prediction on the number of families is lost. A similar procedure might be relevant also for other sixdimensional extensions discussed in [23].

The term $B_{6}$ in eq. (11) remains, even in the unitary gauge, as a Chern-Simons coupling between gauge bosons, and gives rise to dimension-six operators after compactification to $d=4$. These operators are suppressed by a coefficient proportional to the compact volume $R^{2}$. Even though they modify, for instance, the photon couplings, we expect their effects to be negligible because $R \sim$ few $\mathrm{TeV}^{-1}$ [22].

\section{Acknowledgments}

We thank S. Bertolini, L. Bonora and M. Testa for useful discussions. MP also thanks G. Tasinato. This work is partially supported by the European TMR Networks HPRN-CT-2000-00148 and HPRN-CT-2000-00152.
[1] M. B. Green and J. H. Schwarz, Phys. Lett. B 149 (1984) 117.

[2] L. D. Faddeev and S. L. Shatashvili, Phys. Lett. B 167, 225 (1986); N. V. Krasnikov, JETP Lett. 41, 586 (1985) [Pisma Zh. Eksp. Teor. Fiz. 41, 481 (1985)]; O. Babelon, F. A. Schaposnik and C. M. Viallet, Phys. Lett. B 177, 385 (1986); K. Harada and I. Tsutsui, Phys. Lett. B 183, 311 (1987).

[3] J. Preskill, Ann. Phys. 210, 323 (1991).

[4] E. Witten, Phys. Lett. B 117, 324 (1982).

[5] Y.-S. Wu, Phys. Lett. B 153 (1985) 70.

[6] L. Alvarez-Gaumé and P. Ginsparg, Nucl. Phys. B 262 (1985) 439.

[7] C. S. Chu, P. M. Ho and B. Zumino, Nucl. Phys. B 475, 484 (1996) arXiv:hep-th/9602093.

[8] E. Witten, Nucl. Phys. B 223, 422 (1983).

[9] E. Witten, Nucl. Phys. B 223433 (1983).

[10] S. Elitzur and V. P. Nair, Nucl. Phys. B 243, 205 (1984).

[11] B. A. Dobrescu and E. Poppitz, Phys. Rev. Lett. 87, 031801 (2001) arXiv:hep-ph/0102010.

[12] J. Wess and B. Zumino, Phys. Lett. B 37 (1971) 95.

[13] T. Sterling and M. J. Veltman, Nucl. Phys. B 189, 557 (1981); M. S. Chanowitz, M. A. Furman and I. Hinchliffe, Nucl. Phys. B 153 (1979) 402; E. D'Hoker and E. Farhi, Nucl. Phys. B 248, 59 (1984).
[14] G. 't Hooft, lecture given at the Cargèse Summer Institute, 1979, in Recent Developments in Gauge Theories, eds. G. 't Hooft et al. (Plenum, New York, 1980).

[15] D. J. Gross and R. Jackiw, Phys. Rev. D 6 (1972) 477; C. Bouchiat, J. Iliopoulos and P. Meyer, Phys. Lett. B 38, 519 (1972).

[16] M. Dine, N. Seiberg and E. Witten, Nucl. Phys. B 289 (1987) 589.

[17] J. S. Bell and R. Jackiw, Nuovo Cim. A 60 (1969) 47; S. L. Adler, Phys. Rev. 177 (1969) 2426.

[18] R. Jackiw and R. Rajaraman, Phys. Rev. Lett. 54, 1219 (1985) [Erratum-ibid. 54, 2060 (1985)]; R. Rajaraman, Phys. Lett. B 184, 369 (1987); R. Percacci and R. Rajaraman, Int. J. Mod. Phys. A 4 (1989) 4177.

[19] E. Kiritsis, Phys. Lett. B 178, 53 (1986).

[20] R. Percacci, Mod. Phys. Lett. A 12 (1987) 977.

[21] M. Fabbrichesi, M. Piai and G. Tasinato, Phys. Rev. D 64, 116006 (2001) arXiv:hep-ph/0108039, and references therein.

[22] T. Appelquist and B. A. Dobrescu, Phys. Lett. B 516, 85 (2001) arXiv:hep-ph/0106140.

[23] N. Borghini, Y. Gouverneur and M. H. Tytgat Phys. Rev. D 65, 025017 (2002) arXiv:hep-ph/0108094. 